Laser Chem., 1999, Vol. 19, pp. 209-222

Reprints available directly from the publisher Photocopying permitted by license only
(C) 1999 OPA (Overseas Publishers Association) N.V.

Published by license under

the Harwood Academic Publishers imprint,

part of The Gordon and Breach Publishing Group.

Printed in India.

\title{
INFRARED SPECTROSCOPY OF ENZYME REACTION INTERMEDIATES
}

\author{
C. W. WHARTON ${ }^{\mathrm{a}, *}$, M. G. P. PAGE ${ }^{\mathrm{b}}$, R. S. CHITTOCK ${ }^{\mathrm{a}}$, \\ T. E. REGAN ${ }^{\mathrm{a}}$ and S. WARD ${ }^{\mathrm{a}}$ \\ ${ }^{a}$ School of Biochemistry, University of Birmingham, \\ Birmingham B15 2TT, UK \\ ${ }^{\mathrm{b}}$ Preclinical Science, Pharma Division, F. Hoffmann-La Roche Ltd, \\ CH-4070 Basel, Switzerland
}

(Received 18 August 1997)

\begin{abstract}
The serine proteinase mechanism has been studied using a wide range of techniques over many years and is now well understood in terms of the formal chemical changes that occur on the reaction pathway. At the atomic level our understanding is less secure in that available techniques are unable to define interactions such as hydrogen bonding with sufficient accuracy. Atomic interaction is strongly dependent upon separation distances and these need to be measured either directly or indirectly in the dynamic reacting system. Infrared spectroscopy has been applied to the study of chymotrypsin acylenzyme reaction intermediates with the aim of measuring, albeit indirectly, the strength of hydrogen bonding in the oxyanion hole catalytic device. These measurements have been successful with moderately specific substrates but there is a long way to go in terms of improved time-resolution. It is tentatively proposed that tetrahedral intermediates accumulate at high $\mathrm{pH}$. This is, we believe, the first report of the relatively direct observation of this phenomenon in any reacting ester system, chemical or enzymic. The approach used with the serine proteinases has been applied to studies of the transpeptidase of Streptococcus pneumoniae PBP2x. We have shown that the acylenzyme formed from benzylpenicillin hydrogen bonds most strongly in the oxyanion hole. This bonding, in contrast to serine proteinases and $\beta$-lactamases where the interaction facilitates catalysis, serves to stabilise the ester intermediate as required for effective antibiotic action. We see this as a good example of 'Nature knows Best' since semisynthetic antibiotics, designed to be resistant to hydrolysis by $\beta$-lactamases, hydrogen bond more weakly and the acylenzymes hydrolyse more rapidly.
\end{abstract}

Keywords: Proteinase; pencillin; infrared; chymotrypsin; transpeptidase; spectroscopy

${ }^{*}$ Corresponding author. 


\section{INTRODUCTION}

\section{The Mechanism of Serine Proteinase Catalysis}

The serine proteinase enzymes form a disparate family that encompasses the digestive enzymes chymotrypsin and trypsin, thrombin, which is involved in blood clotting, as well as bacterial enzymes such as subtilisin. The structures of the mammalian enzymes fall into one class and are composed predominantly of $\beta$-sheet, while the bacterial enzymes are predominantly $\alpha$-helical. Despite this difference in structure all serine proteinases have an essentially identical catalytic apparatus and associated mechanism [1]. This is comprised of the relay system [Asp-His-Ser], the oxyanion hole and a specificity conferring sidechain binding pocket. The serine of the relay system acts as a nucleophile which attacks the substrate at the susceptible carbonyl group, where the peptide chain is cleaved. The His residue, strongly hydrogen bonded to the Ser acts as a general base to remove a proton from the Ser or water in both steps of the reaction (see below) and to provide a proton, while acting as an acid, for the leaving nitrogen atom in acylation or the serine in deacylation. The Asp residue serves to stabilise the conformation of the His residue as required for its action as a general base. The oxyanion hole is comprised of two backbone amide nitrogens, whose hydrogens are so placed to hydrogen bond to the susceptible carbonyl that is to be attacked by the serine residue. The predefined spatial organisation of these amide hydrogens ensures that there is no entropy requirement upon substrate or acyl group interaction. The oxyanion hole has been supposed to stabilise the negative charge which develops in the transition states for each of the two steps of the enzyme-catalysed reaction:

$$
\mathrm{E}+\mathrm{S} \stackrel{\mathrm{K}_{\mathrm{s}}}{\leftrightarrow} \mathrm{ES} \stackrel{k_{2}}{\rightarrow} \mathbf{E A}+\mathbf{P}_{1} \underset{\mathrm{H}_{2} \mathrm{O}}{\stackrel{k_{3}}{\rightarrow}} \mathrm{E}+\mathbf{P}_{2}
$$

where EA is the acylenzyme. The C-terminal part of the substrate is lost in the acylation step, characterised by $k_{2}$, while the $\mathrm{N}$-terminal part is lost as a consequence of deacylation, characterised by $k_{3}$. The acylation step generates a unique ester bond in the structure of the covalently bonded enzyme substrate complex. The deacylation step 
represents a hydrolysis of the ester intermediate. The acylation and deacylation steps are almost mirror images of one another. The amide bonds of proteins or peptides are hydrolytically resistant and a complex mechanism has evolved to cope with this. A simplified version of the mechanism is shown in Figure 1 (for more detail, see Ref. [1]). Note that putative tetrahedral intermediates have been omitted.

The unique ester group of the acylenzyme is amenable to study using IR spectroscopy since it absorbs at a frequency that is higher than the peptide amide groups, although ${ }^{13} \mathrm{C}=\mathrm{O}$ or ${ }^{13} \mathrm{C}={ }^{18} \mathrm{O}$ isotopic labelling, with associated down-frequency shifts of 40 and $80 \mathrm{~cm}^{-1}$, is required to ensure band assignment, since perturbation of the protein spectrum by formation of the acylenzyme can, particularly at low $\mathrm{pH}$, interfere with the ester band(s) [2-6].

Questions of importance that may be addressed by IR spectroscopy in attempting to understand how this molecular machine functions include:

How strongly is the acyl carbonyl group hydrogen bonded into the oxyanion hole in the acylenzyme? Can the hydrogen bonding provide ground state strain and so aid the catalytic mechanism? Does the carbonyl group have a single orientation in the enzyme active site that is 'productively' oriented for catalysis? Is the orientation of the carbonyl group influenced by the kinetic specificity of the acyl group? Are more specific groups better oriented by stronger hydrogen bonding to provide a more efficient entry into the reaction channel? Are there significant conformational differences seen in the X-ray crystallographic structures and those deduced in solution from IR (or NMR)

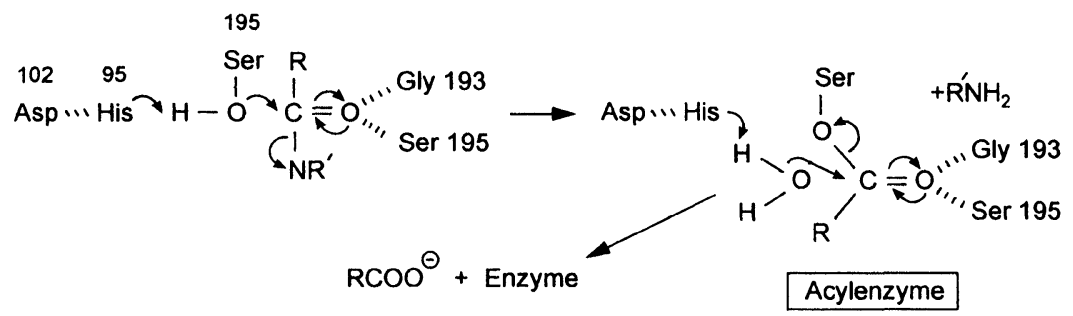

FIGURE 1 A simplified version of the serine proteinase mechanism in which putative tetrahedral intermediates are omitted. The acylation step is rate-limiting for amide and peptide hydrolysis, while deacylation is rate-limiting for ester hydrolysis. Thus the acylenzyme accumulates only during ester hydrolysis. 
spectra and if so how do they relate to the conditions (e.g., $\mathrm{pH})$ in which the experiments were carried out? Does the conformational balance shift or hydrogen bonding strengthen as the $\mathrm{pH}$ is raised across the $\mathrm{pKa}$ of 7.0 which governs enzyme activity? Is a particular active conformation selected?

\section{IR Spectroscopy of Serine Proteinase Acylenzymes}

The structures of the two acyl groups whose difference spectra are shown in Figure 3 are given in Figure 2. As shown in Figure 3 the cinnamoyl group in cinnamoyl-chymotrypsin is seen to exist in two conformations in the active centre, while $o-\mathrm{OH}, \alpha$-methylcinnamoylchymotrypsin which exists in a single conformation in the crystalline state [7] also shows two conformations in IR spectra. There is thus a clear difference between the solution and crystalline states. One conformation is proposed to be productive in that the carbonyl group is bonded into the oxyanion hole, while the other is nonproductive with the carbonyl oxygen pointing away from the oxyanion hole as seen in the X-ray structure [2,7]. These conformations are shown schematically in Figure 4. In contrast to the cinnamoyl-group the more flexible saturated acyl group, $\beta$-phenylpropionyl-, see Figures 2 and 3, exists in a single but poorly defined conformation in the acylchymotrypsin. That the conformation is poorly defined is shown by the broadness of the band which is proportional to the structural or motional dispersity of the bound acyl group. This reflects primarily its flexibility since it is a ten-fold better substrate than is the cinnamoylgroup. This contrasts with the ability of the rigid planar cinnamoyl group to flip between two well defined positions.

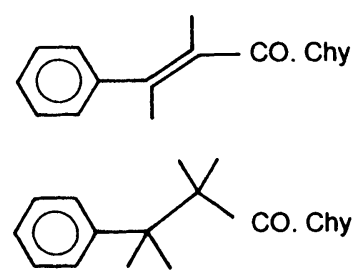

FIGURE 2 The cinnamoyl (top) and $\beta$-phenylpropionyl (bottom) acyl groups. The former is rigid and planar while the latter has more rotational freedom. 

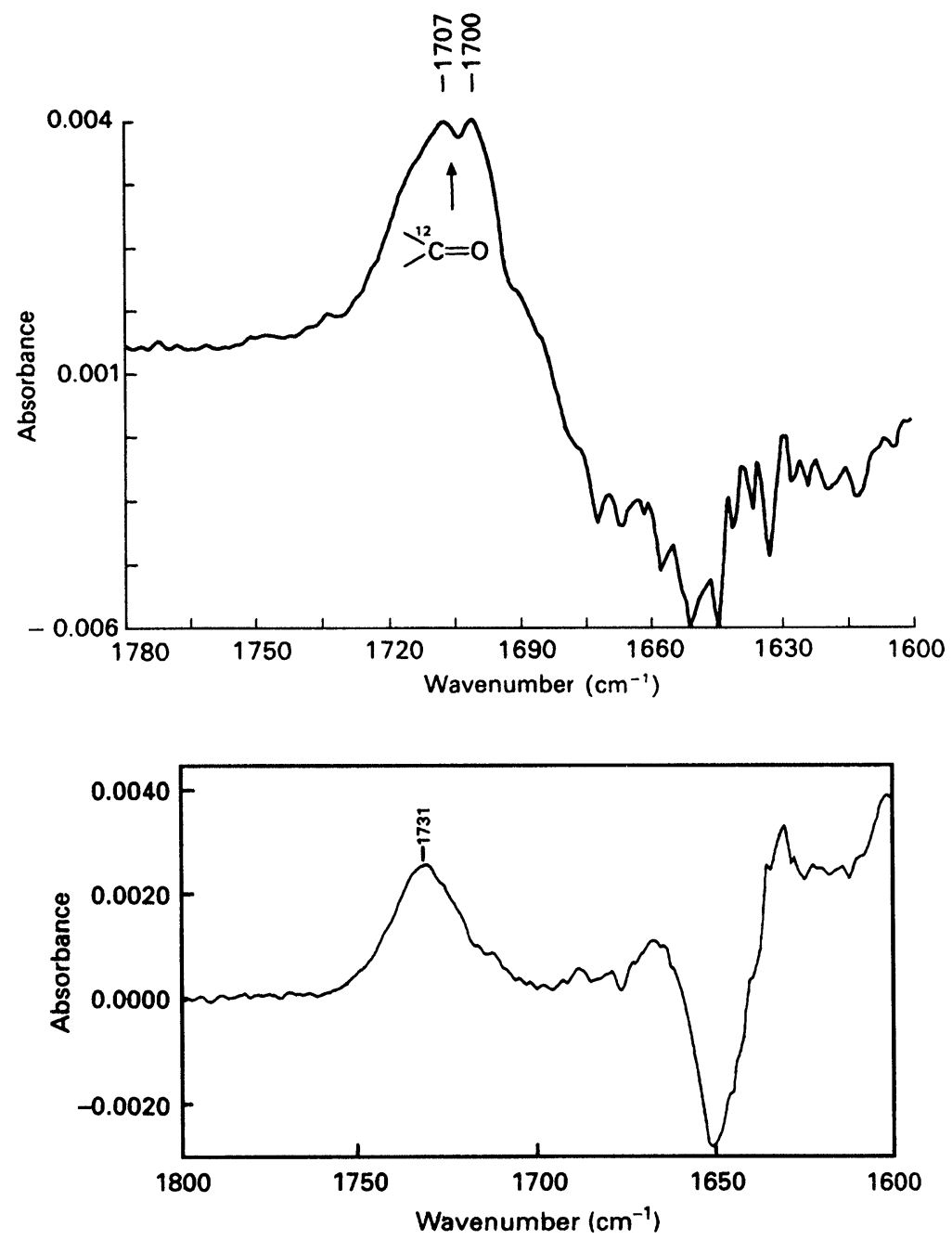

FIGURE 3 Isotope-edited IR difference spectra of cinnamoyl-chymotrypsin (top) and $\beta$-phenylpropionyl-chymotrysin (bottom) at pM 4.0. In each case the heavy atom substituted version of the acylenzyme was subtracted from the unedited version. Thus for the cinnamoyl group the spectrum represents ${ }^{12} \mathrm{C}={ }^{16} \mathrm{O}$ minus ${ }^{13} \mathrm{C}={ }^{16} \mathrm{O}$ giving a $40 \mathrm{~cm}^{-1}$ down-frequency shift for the carbonyl absorbance, while for the $\beta$ phenylpropionyl group the spectrum represents ${ }^{12} \mathrm{C}={ }^{16} \mathrm{O}$ minus ${ }^{13} \mathrm{C}={ }^{18} \mathrm{O}$, giving an $80 \mathrm{~cm}^{-1}$ down-frequency shift. Spectra were collected using Nicolet $60 \mathrm{SX}$ and Bruker IFS66 spectrometers with a $50 \mu \mathrm{m}$ pathlength cell with $\mathrm{CaF}_{2}$ windows. Concentration of acylenzymes was $1.2 \mathrm{mM}$. All measurements were made in deuterium oxide. 

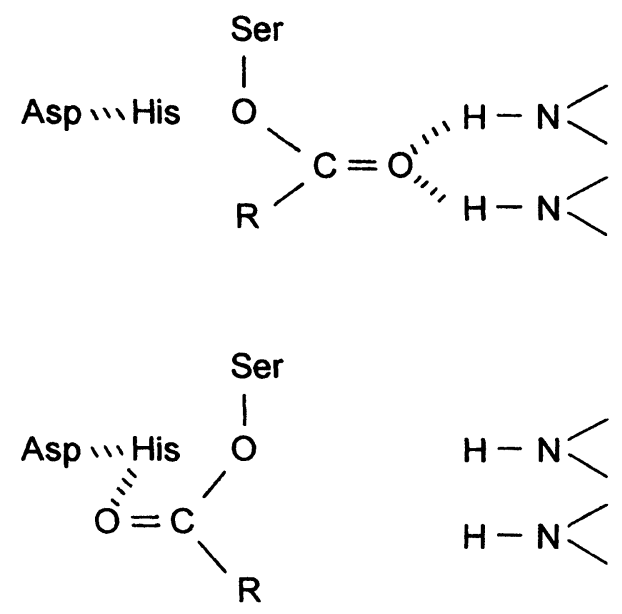

FIGURE 4 A schematic representation of the productive (nominally reactive) and nonproductive conformations of cinnamoyl-chymotrypsin.

The IR frequencies of the various conformers have been analysed in terms of hydrogen bonding in the oxyanion hole. This has been achieved by a combination of studies of simple model esters in a variety of solvents and some fairly simple calculations. A calculation that makes use of the linear energy of the wavenumber scale and the bond strength and one in which the bond is analysed in terms of simple harmonic motion give good agreement [2]. The productive conformer of cinnamoyl-chymotrypsin is hydrogen bonded somewhat more strongly to the oxyanion hole than it is to water, while the nonproductive conformation and the $\beta$-phenylpropionyl group are poorly hydrogen bonded.

Studies of more specific acyl groups in the form of CBZ-gly- and gly-gly-, that can form hydrogen bonds with the active site of the enzyme via the substrate $\mathrm{C}=\mathrm{O}$ and $\mathrm{N}-\mathrm{H}$ groups, reveal in each case that a single conformation exists, but better defined than that of the $\beta$ phenylpropionyl group [9]. At low $\mathrm{pH}$, where the enzyme is inactive, the acylcarbonyl frequency is very high at approximately $1760 \mathrm{~cm}^{-1}$ but this decreases and the hydrogen bonding gets stronger as the $\mathrm{pH}$ is raised and the enzyme becomes active. The frequency decreases by 
$15 \mathrm{~cm}^{-1}$ as the $\mathrm{pH}$ is raised and this is estimated to represent an increase of $5 \mathrm{~kJ} / \mathrm{mole}$ in hydrogen bonding. Notably the intensity of the carbonyl IR absorbance becomes low at high $\mathrm{pH}$, being only $15 \%$ of that at low $\mathrm{pH}$ and the absorption is also very broad. We very tentatively assign this loss of absorbance, which has proved repeatable in several systems, to tetrahedral intermediate formation. No such proposal has been previously put forward but this is not surprising since IR and Raman spectroscopies are the only methods of detecting such a phenomenon. Bender used IR spectroscopy to very clearly demonstrate tetrahedral intermediate formation by loss of carbonyl absorbance in the reaction of alkoxide ions with ethyl trifluoroacetate [8]. Surprisingly the approach does not seem to have been much followed up, although dynamic measurements would be required to decisively demonstrate involvement on the reaction pathway.

Complete reaction courses covering a period of $10 \mathrm{sec}$ have been measured, where the first spectrum, obtained by averaging 20 stoppedflow experiments is obtained in $60 \mathrm{~ms}$ [6, 9]. These studies are progressing towards ever more specific substrates e.g., gly-gly-L-proL-Phe-chy with a deacylation rate constant at high $\mathrm{pH}$ of $100 \mathrm{~s}^{-1}$. FTIR will not be capable of the speed required to measure these reactions so a single frequency laser or dispersive IR method will be necessary.

\section{$\beta$-Lactamases and Transpeptidases}

The former enzymes are responsible for resistance to $\beta$-lactam antibiotics since they catalyse the hydrolytic opening of the lactam ring, see Figure 5, often very efficiently. There are many such enzymes and each type of bacterium has its own complement of enzymes with varying specificity towards particular antibiotics, which confers upon it a specific pattern of resistance to antibiotics [10]. These enzymes also work by the serine acylenzyme mechanism but the components other than active serine and an oxyanion hole are quite different from the serine proteinases [10]. Thus, although the general base which activates the active serine has not been conclusively identified, a glutamic acid, a lysine or a tyrosine residue are possible candidates for this role, with Glu-166 the favourite. The D,D-transpeptidase enzymes, the targets of antibiotic action, are inhibited by the $\beta$-lactam antibiotics in the form 


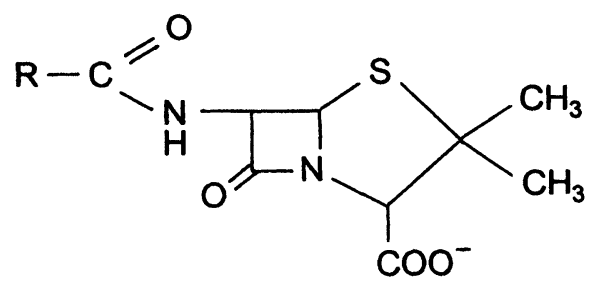

FIGURE 5 The structure of the penicillin ring framework. The R group is benzyl in the case of the original natural penicillin benzylpenicillin and 2-chlorophenyl, 5methylisoxazolyl for cloxacillin.

of stable acylenzymes. This inhibition prevents the cross-linking of the cell wall of the bacteria and renders them unable to cope with the osmotic pressure of a dilute aqueous environment. This is how these antibiotics kill bacteria, so the transpeptidases represent the 'killing' targets.

Both types of enzyme form acylenzymes, so it is happily the case that the IR methods developed for the study of the serine proteinases can be directly adopted for studies of $\beta$-lactamases and transpeptidases. These studies may be able to contribute to a better understanding of how these two classes of enzyme, which have much in common in terms of structure and it is believed, in function, may be distinguished in terms of substrate/inhibitor binding and catalysis. Such a distinction, in terms of conformational analysis of interaction with the oxyanion hole, may provide potent information which could be used to inform the drug design process. Effective antibiotic action might be achieved by the design of a single molecule which would be immune to lactamases but inhibit transpeptidase or one compound which inhibits both enzymes. Alternatively, and probably more effectively, two compounds might be used, each targeted towards one of the enzymes [11].

The penicillin target proteins are membrane anchored in vivo but have been genetically manipulated to remove the anchor. The resulting overexpressed proteins are soluble and can be used exactly as with the serine proteinases. We have studied the interaction of Streptococcus pneumoniae $\mathrm{PBP} 2 \mathrm{x}$ enzyme with a range of antibiotics as described below. 


\section{The Role of IR Spectroscopy}

In 1980 Belasco and Knowles showed that IR spectroscopy could be applied to the study of antibiotic interaction with $\beta$-lactamase [12]. They demonstrated that cefitoxin, an antibiotic that is slowly turned over by the lactamase RTEM from $E$. coli, forms a relatively stable acylenzyme whose spectrum is characterised by an acyl carbonyl band with a maximum at $1753 \mathrm{~cm}^{-1}$, well resolved from the protein background. This band was also well separated from the $\beta$-lactam absorbance at $1767 \mathrm{~cm}^{-1}$. The $E$. coli $\beta$-lactamase TEM3 used in our studies (see below) does not catalyse the hydrolysis of cefitoxin so we have been unable to compare the acylenzymes formed with the two lactamases.

Generally IR spectroscopy falls nicely between the 'high structure' methods of X-ray crystallography and NMR and the 'low structure' but high time-resolution $\mathrm{UV} /$ visible spectroscopies. It is possible to obtain useful structural information in time-resolved mode by using stopped-flow mixing. It is thus possible to study catalysis and reactions of these enzymes in 'active' conditions. Most particularly IR spectroscopy is a 'fast' method by comparison with X-ray crystallography and NMR and may also help to speed the evolution of drug design by providing structural information rapidly.

\section{Anthraniloyl- $\beta$-Lactamase (TEM3)}

TEM3 is a $\beta$-lactamase from $E$. coli. which can be acylated with isatoic anhydride to give the anthraniloyl (o-aminobenzoyl)-acylenzyme intermediate. Isatoic anhydride is a good inhibitor of chymotrypsin, where loss of $\mathrm{CO}_{2}$ on acylenzyme formation drives the reaction [13]. Anthraniloyl-chymotrypsin deacylates extremely slowly but anthraniloyl-TEM3 deacylates over a period of a few hours at neutral $\mathrm{pH}$, see Figure 6. A feature seen at $1690 \mathrm{~cm}^{-1}$ and which decays with time is believed to represent the acylenzyme carbonyl group, although this cannot be assigned with certainty until such time as the ${ }^{13} \mathrm{C}=\mathrm{O}$ isotope edited difference spectrum is available. In the serine proteinases, protein perturbation features appear at this frequency, probably arising from perturbation of $\beta$-structure at the active centre. Features near 1660 and $1620 \mathrm{~cm}^{-1}$ represent protein structural perturbation. 


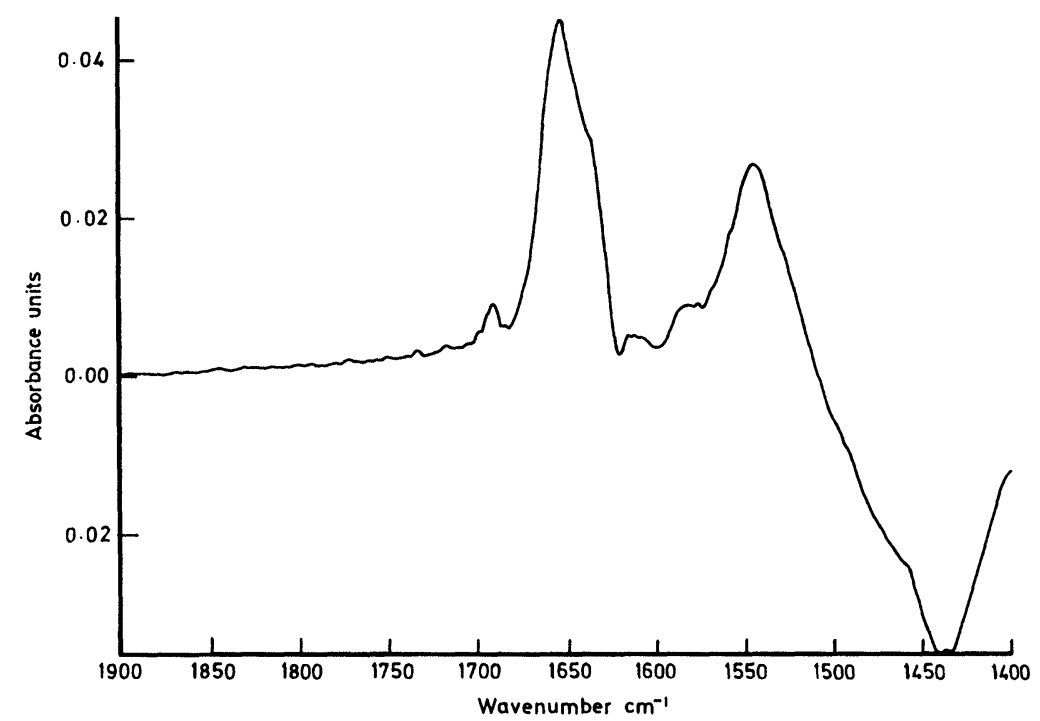

FIGURE 6 Infrared difference spectrum of isatoyl (anthraniloyl) TEM3 $\beta$-lactamase from E. coli. In this spectrum the free enzyme was subtracted from the spectrum of the ${ }^{12} \mathrm{C}={ }^{16} \mathrm{O}$ inhibitor. The pM was 7.6 and the concentration of enzyme $0.7 \mathrm{mM}$. Conditions otherwise as in Figure 3.

Some of this arises as a consequence of the extensive $\mathrm{H} \rightarrow \mathrm{D}$ exchange (seen at $1545 \mathrm{~cm}^{-1}(\mathrm{H}) \rightarrow 1440 \mathrm{~cm}^{-1}$ (D)) that is stimulated by the deacylation reaction. This $\mathrm{H} \rightarrow \mathrm{D}$ exchange is proposed to arise from exposure of internal, normally nonexchangeable protons during the catalytic process and implies that there must be a substantial structural change during the deacylation process that allows access to the interior core of the protein. The enzyme was incubated for $72 \mathrm{~h}$ with deuterium oxide prior to the experiment so the residual core of protons is resistant to exchange in the resting enzyme.

\section{Hydrogen Bonding in the Oxyanion Hole}

The frequency of the presumed acyl carbonyl group may be compared with that of ethyl anthranilate, a model for the ester bond of the acyl enzyme. The frequency is dependent upon the solvent and occurs at $1693 \mathrm{~cm}^{-1}$ in ethanol. This suggests that hydrogen bonding in the oxyanion hole is less strong than in water, which shifts the frequency 
down to $1679 \mathrm{~cm}^{-1}$. This, in turn, suggests that the relatively nonspecific nature of the anthraniloyl group renders it incapable of hydrogen bonding strongly in the oxyanion hole. A preliminary analysis indicates that there is only a single conformation of the acyl group but conformation analysis will have to await isotope editing.

\section{Acylenzymes of Streptomyces Pneumoniae PBP2X}

Penicillin antibiotics form stable acylenzymes with the target penicillin binding proteins (PBP's). Benzylpenicillin, the original Natural antibiotic, reacts rapidly with PBP's but is hydrolysed extremely rapidly by $\beta$-lactamases and so is ineffective as an antibiotic. Many attempts have been made to circumvent the resistance problem by using semisynthetic penicillins (e.g., ampicillin, cloxacillin, methicillin) where the amido-sidechain $\mathbf{R}$ group has been altered to reduce the susceptibility to hydrolysis by $\beta$-lactamases. Considerable success has been achieved but the effectiveness of these antibiotics has been continuously eroded by the appearance of new mutant forms of $\beta$ lactamase that can effectively hydrolyse these semi-synthetic antibiotics. We have measured difference spectra of a number of acylPBP's, two of which are shown in Figure 7, together with spectra of the antibiotics (both the same in the 1700 to $1800 \mathrm{~cm}^{-1}$ region since the lactam rings are identical in each case) and the ethyl ester models of the acylenzymes, also both the same in this spectral region for the reason given above. The reason why the spectra of the antibiotics and the models are the same for the two antibiotics is that the sidechain part of the molecules, where the difference lies, occurs at lower frequencies at ca. $1640 \mathrm{~cm}^{-1}$.

A clear difference is seen between the acylenzymes with the benzylpenicillin version having a lower frequency than the semisynthetic cloxacillin. Also notable is the narrower bandwidth of the benzylpenicillin absorbance indicating a well ordered structure, when compared with both cloxacillin and the model ethyl ester. Hydrogen bonding in the oxyanion hole is, in both cases, stronger than in deuterium oxide, with the benzylpenicillin version being particularly strong and well defined. Inspection of the band profile for the cloxacillin acylenzyme reveals that there may be two conformations present. One of these has an absorbance frequency lower than 


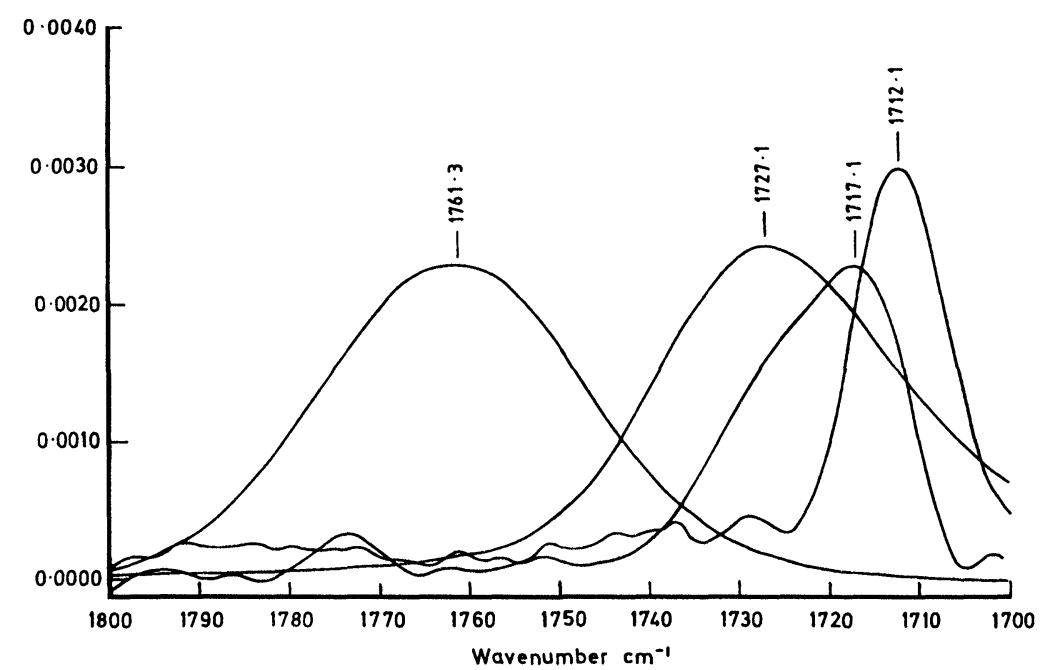

FIGURE 7 Infrared difference spectra of (left to right) benzylpenicillin and cloxacillin, the ethyl esters of the ring opened antibiotics, Strep. pneumoniae PBP2x acylenzyme with cloxacillin and the equivalent with benzylpenicillin. The antibiotics and ethyl esters were $5 \mathrm{mM}$ in deuterium oxide, while the acylenzymes were $0.8 \mathrm{mM}$. The intensities of the antibiotic and ester spectra have been scaled to approximately match those of the acylenzymes.

deuterium oxide while the other appears to coincide with the model frequency. It is thus proposed that the higher frequency component arises from a conformer in which the carbonyl group is exposed to solvent and thus in a nonproductive conformation.

These results, and others not shown, suggest to us that the maxim 'Nature knows best' is reflected in these observations or at least one facet thereof. On the one hand the natural antibiotic is perfectly tuned to block the target PBP's and yet is hydrolysed most rapidly by $\beta$ lactamases. Evolution has had more time and opportunity to tune these enzyme-substrate/inhibitor systems compared with cloxacillin, which does neither job very well but is a somewhat more effective compromise. These concepts nicely illustrate how difficult it is to 'beat Nature at her own game' and mean that a very fundamental understanding of molecular interactions will be required for the deliberate design of new pharmaceuticals required to be effective in an embattled environment. Not surprisingly many pharmaceutical 
organisations have tackled the problem of antibiotic resistance by using a bifurcated approach in which an inhibitor for each enzyme is supplied. The telling outcome is that again natural compounds, discovered essentially by accident, have proved most effective.

In view of the above and many other findings we need to adopt a suitably humble attitude in our stumbling attempts to manipulate Nature. We hope that, in the future, infrared spectroscopy will add to the other physical, biochemical and genetic techniques available, to allow us to pursue a more deterministic path to drug discovery. Perhaps the most potent combination will be the use of genomics for target identification followed by structural and computational methods coupled with combinatorial chemistry/biochemistry. Rapid conformational analysis by IR spectroscopy seems likely to be able to fill in an important niche in the overall process.

\section{Acknowledgements}

This work has been supported by funding from BBSRC (UK) and F. Hoffmann-La Roche, Basel, Switzerland.

\section{References}

[1] Wharton, C. W. (1997). The Serine Proteinases in Comprehensive Biological Catalysis (Ed. Sinnott, M. L.) Academic Press, London. Vol. 1, Chapter 9.

[2] White, A. J. and Wharton, C. W. (1990). Hydrogen bonding in chymotrypsin catalysis, Biochem. J., 270, 627-337.

[3] Tonge, P. J., Pusztai, M., White, A. J., Wharton, C. W. and Carey, P. R. (1991). Resonance Raman and FTIR spectroscopic studies of the acyl carbonyl group in 3(5-methyl-2-thienyl)acryoyl-chymotrypsin, Biochemistry, 30, 4790-4795.

[4] White, A. J., Drabble, K, Ward, S. and Wharton, C. W. (1992). The analysis and elimination of protein perturbation in FTIR difference spectra of acylchymotrypsins, Biochem. J., 287, 317-323.

[5] Johal, S. S., White, A. J. and Wharton, C. W. (1994). The effect of specificity on ligand conformation in acylchymotrypsins, Biochem. J., 297, 281-287.

[6] White, A. J., Drabble, K. and Wharton, C. W. (1995). A stopped-flow apparatus for infrared spectroscopy of aqueous solutions, Biochem. J., 306, 843-849.

[7] Stoddard, B. L., Bruhnke, J., Porter, N. A., Ringe, D. and Petsko, G. (1990). Structure and activity of two photoreversible cinnamates bound to chymotrypsin, Biochemistry, 29, 4871-4879.

[8] Bender, M. L. (1953). Intermediates in the reactions of carboxylic acid derivatives II, J. Am. Chem. Soc., 75, 5986-5990.

[9] Regan, T. (1997). Ph.D. Thesis, University of Birmingham, Birmingham, U.K.

[10] Jamin, M., Wilkin, J.-M. and Frere, J.-M. (1995). Bacterial DD-transpeptidases and penicillin, In: Essays in Biochemistry, Eds. Apps, D. K. and Tipton, K. F., Portland Press, London, U.K. 
[11] Richter, H. G. F., Angehrn, P., Hubschwerien, C., Kania, M., Page, M. P. G., Specklin, J.-L. and Winkler, F. K. (1996). Design, synthesis and evaluation of $2 \beta$ alkenyl penam sulphone acids as inhibitors of $\beta$-lactamases. J. Med. Chem., 39, $3712-3722$.

[12] Fisher, J., Belasco, J. G., Khosla, S. and Knowles, J. R. (1980). $\beta$-Lactamase proceeds via an acyl-enzyme intermediate, Biochemistry, 19, 2895-2901.

[13] Moorman, A. R. and Abeles, D. H. (1982). Inhibition of chymotrypsin by isatoic anhydride, J. Am. Chem. Soc., 104, 6786-6787. 